\title{
Estudio de los factores que afectan la estabilidad de nanofluidos usando un diseño factorial fraccionado
}

\section{Study of factors that affect nanofluids stability using a fractional factorial design}

\author{
K. Cacua Maderoํㅜㄹ R. Buitrago-Sierra² ${ }^{2}$ E. Pabón Gelves ${ }^{3}$, \\ B. Herrera Múnera ${ }^{4}$, J. L. Rentería ${ }^{5}$
}

Recibido: 16 de diciembre 2016

Aceptado: 02 de junio 2017

\section{Resumen}

A nivel mundial la búsqueda de alternativas que permitan incrementar la eficiencia energética en los procesos industriales ha estado en constante crecimiento, debido principalmente a la disminución en las reservas energéticas no renovables y a la preocupación mundial por la disminución de los gases de efecto invernadero.

1 Grupo Materiales Avanzados y Energía, Facultad de Ingenierías, Instituto Tecnológico Metropolitano. Grupo de Ciencia de los Materiales Avanzados, Facultad de Ciencias, Universidad Nacional de Colombia, Sede Medellín

karencacua@itm.edu.co,kpcacuam@unal.edu.co

2 Grupo Materiales Avanzados y Energía, Facultad de Ingenierías, Instituto Tecnológico Metropolitano robinsonbuitrago@itm.edu.co

3 Grupo de Ciencia de los Materiales Avanzados, Facultad de Ciencias, Universidad Nacional de Colombia, Sede Medellín

epabon@unal.edu.co

4 Grupo Materiales Avanzados y Energía, Facultad de Ingenierías, Instituto Tecnológico Metropolitano bernardoherrera@itm.edu.co

5 Joven Investigador ITM, Estudiante de Ingeniería Electromecánica

Grupo Materiales Avanzados y Energía, Facultad de Ingenierías, Instituto Tecnológico Metropolitano jorgerenteria137111@correo.itm.edu.co 
La mayoría de los procesos a nivel industrial, involucran producción o absorción de energía en forma de calor, estos procesos podrían tener mayores eficiencias energéticas, a partir de la sustitución de sus fluidos térmicos convencionales (agua, etilenglicol, aceite motor) por nanofluidos. Los nanofluidos son suspensiones coloidales obtenidas al dispersar nanopartículas (tamaño<100 $\mathrm{nm}$ ) de diferentes materiales, una de sus principales aplicaciones es en transferencia de calor y se usan con el objetivo de incrementar la conductividad térmica de los fluidos convencionales utilizados en estos procesos. Sin embargo, a pesar de los numerosos estudios que se encuentran en la literatura mostrando los incrementos en la conductividad térmica y la transferencia de calor en los diferentes sistemas térmicos a escala de laboratorio, la inestabilidad de los nanofluidos ocasionada por la aglomeración y sedimentación de las nanopartículas sigue siendo un reto de investigación.

Entre los factores que afectan la estabilidad de un nanofluido se encuentran el método de dispersión, la concentración de nanopartículas, el pH, tipo de nanopartícula y aditivos químicos. En este estudio, se prepararon nanofluidos de óxido de aluminio $\left(\mathrm{Al}_{2} \mathrm{O}_{3}\right)$ y dióxido de titanio $\left(\mathrm{TiO}_{2}\right)$ y se implementó un diseño factorial fraccionado $2^{6-2}$ de resolución IV, con el fin de evaluar el efecto de diferentes parámetros en la estabilidad de los nanofluidos. La estabilidad se evaluó como el cambio en la conductividad térmica con el tiempo. En el estudio estadístico realizado, los factores e interacciones con un efecto significativo en la estabilidad del nanofluido fueron:el tiempo de ultrasonido y la concentración de surfactante, tipo de nanopartícula/tipo de surfactante y tipo de nanopartícula/concentración de surfactante respectivamente.

Palabras clave: nanofluidos, estabilidad, conductividad térmica, diseño experimental factorial fraccionado

\section{Abstract}

Worldwide searches for alternatives in order to increase energy efficiency in industrial processes has been steadily growing, mainly due to the decrease in non-renewable energy reverses and concerns for the reduction of greenhouse gases.

Most processes at industrial level, produce or involve energy absorption as heat, these processes may have greater energy efficiency, from replacing their conventional thermal fluids (water, ethylene glycol, engine oil) by nanofluids. Nanofluids are colloidal suspensions obtained by dispersing nanoparticles (particle size $<100 \mathrm{~nm}$ ) of different materials, one of its main applications is in heat transfer and are used in order to increase the thermal conductivity of conventional fluids used in these processes. However, despite numerous studies in the literature showing increases in thermal conductivity and heat transfer in the various thermal systems at laboratory scale, instability nanofluids caused by agglomeration and sedimentation of nanoparticles remains a research challenge.

Among the factors affecting the stability of nanofluids are dispersion method, nanoparticle concentration, $\mathrm{pH}$, type of nanoparticle and chemical additives. In this study, nanofluids of 
aluminum oxide $\left(\mathrm{Al}_{2} \mathrm{O}_{3}\right)$ and titanium dioxide $\left(\mathrm{T}_{i} \mathrm{O}_{2}\right)$ were prepared and a fractional factorial design $2^{6-2}$, resolution $I V$, was implemented, in order to evaluate the effect of different parameters on the stability of nanofluids. Stability was evaluated as the change in thermal conductivity with time. In the statistical study performed, factors with a significant effect on the stability of nanofluid were: ultrasonic time and surfactant concentration, as well as the interactions nanoparticle type/surfactant type and nanoparticle type/surfactant concentration.

Keywords: nanofluids, stability, thermal conductivity, fractional factorial experimental design

\section{Introducción}

os nanofluidos actualmente son conocidos como suspensiones obtenidas Lal dispersar nanopartículas (tamaño<100 nm) con bajas concentraciones volumétricas (generalmente menores al 10\%) en fluidos base como agua, etilenglicol, aceites y soluciones poliméricas ${ }^{[1]}$. El principal objetivo de las nanopartículas suspendidas en un fluido es incrementar la conducción de calor debido a su alta área superficial e incrementar la conductividad térmica de los fluidos ${ }^{[2]}$.

A partir de la introducción de los nanofluidos para aplicaciones de transferencia de calor realizada por Choi et al. ${ }^{[3]}$, se han utilizado nanopartículas de diferente naturaleza tales como metales (ej. Oro, cobre), óxidos metálicos (ej. Sílica, alúmina, zirconia, titania), carburos metálicos (ej. SiC), nitruros metálicos (ej. AIN, SiN), carbón en varias formas (ej. Diamante, grafito, nanotubos de carbono, fullerenos), así como también nanopartículas mezcladas o compuestas ${ }^{[4]}$.

Sin embargo, a pesar de todo el potencial que tienen los nanofluidos para incrementar la eficiencia energética de los sistemas de transferencia de calor, estos aún no han podido tener aplicación industrial, debido principalmente a problemas de inestabilidad (variación de sus propiedades termofísicas con el tiempo debido a la agregación y sedimentación de nanopartículas) [4]-[10]. Es por esto que estudiar los factores que afectan la estabilidad y encontrar métodos que permitan obtener nanofluidos estables, para aplicaciones de transferencia de calor, se ha convertido en uno de los principales retos de investigación a nivel mundial ${ }^{[4]-[10]}$ La evaluación de la estabilidad de un nanofluido puede estudiarse por diferentes métodos entre los que se encuentran la variación de la absorbancia UV-visible (la concentración de nanopartí- 
culas en suspensión es proporcional a la absorbancia), potencial zeta (indica el grado de repulsión entre partículas adyacentes con carga similar suspendidas en un fluido), variación de distribución de tamaño con el tiempo por dispersión de luz dinámica (DLS), inspección visual [11],[12] y la variación en sus propiedades termofísicas ${ }^{[13]}$ entre otras.

Entre los diferentes factores que afectan la estabilidad se encuentran el tipo de nanopartícula utilizada, su concentración, el tipo y concentración de surfactante, tiempo de ultrasonido y potencia de ultrasonido y el pH entre otros factores [14], [15]. Sin embargo, aunque se han encontrado diferentes estudios que evalúan el efecto de los diferentes factores en diferentes nanofluidos [10], [16], [17], en la literatura revisada no se encontró un estudio experimental que analizara el efecto de más de cinco factores en la estabilidad de nanofluidos de óxidos metálicos. Cuando el número de factores se incrementa ( 5 o más) es común utilizar los diseños factoriales fraccionados, los cuáles utilizan menos corridas experimentales que los diseños factores completos y permiten identificar los factores o sus interacciones que tienen una influencia significativa en una variable respuesta. Este tipo de diseños es utilizado en las primeras etapas de experimentación y los factores o interacciones identificados cómo importantes se estudian con más profundidad en estudios posteriores ${ }^{[18]}$

En este estudio experimental se implementó un diseño factorial fraccionado $2^{6-2}$ de resolución IV (los efectos principales se confunden con interacciones de tres o más factores y las interacciones dobles están confundidas entre sí), con el fin de determinar los factores o sus interacciones que tuvieran un efecto significativo en la estabilidad de nanofluidos de óxido de titanio $\left(\mathrm{T}_{1} \mathrm{O}_{2}\right)$ y óxido de aluminio $\left(\mathrm{Al}_{2} \mathrm{O}_{3}\right)$, con el fin de investigar posteriormente dichos factores o interacciones y optimizar sus valores para obtener nanofluidos estables.

\section{Materiales y métodos}

Los nanofluidos se prepararon por el método de dos pasos, el cual consiste en adicionar las nanopartículas sólidas y el surfactante al fluido base y dispersarlas usando una sonda de ultrasonido (Qsonica-Q500). Para la disipación de calor en el nanofluido durante la sonicación, se utilizó un baño termostatado (LAUDA, RA24). El montaje experimental se muestra en la FI- 
GURA 1. Se utilizaron nanopartículas de óxido de Aluminio $\left(\mathrm{Al}_{2} \mathrm{O}_{3}\right.$, fase gama, $<50 \mathrm{~nm})$ y dióxido de titanio $\left(\mathrm{T}_{\mathrm{i}} \mathrm{O}_{2},<21 \mathrm{~nm}\right)$, obtenidas comercialmente en Sigma-Aldrich. Los surfactantes utilizados fueron Dodecilbenceno Sulfonato de Sodio (SDBS, por su nombre en inglés), de tipo aniónico y Bromuro de hexadeciltrimetilamonio (CTAB, por su nombre en inglés) de tipo catiónico.

La estabilidad del nanofluido se determinó teniendo en cuenta la variación de la conductividad térmica con el tiempo, utilizando un analizador transitorio de hilo caliente KD2 Pro (Decagon, Inc), este dispositivo cuenta con una aguja de $6 \mathrm{~cm}$ de longitud y un diámetro de $1,3 \mathrm{~mm}$, la cual incluye un sensor de temperatura y un medio de calentamiento. La aguja se inserta en el fluido y una vez se estabilice la temperatura del medio y la aguja, se inicia la medida La conductividad térmica se midió inmediatamente después de preparados los nanofluidos y ocho días después de dejarlos en reposo. Para evaluar el efecto de los diferentes factores y sus interacciones, se implementó un diseño factorial fraccionado $2^{[6-2]}$ de resolución IV, realizando 16 corridas experimentales. En la Tabla 1 se muestran los diferentes factores estudiados y se establecen sus niveles. Los experimentos fueron realizados en orden aleatorio, con el objetivo de que el error asociado a factores no controlados se reparta de forma homogénea en todos los experimentos, la matriz de diseño ejecutada se muestra en la Tabla 2. Los resultados obtenidos se procesaron utilizando el software estadístico Minitab ${ }^{\circledR} 17$ versión demo ${ }^{[19]}$.

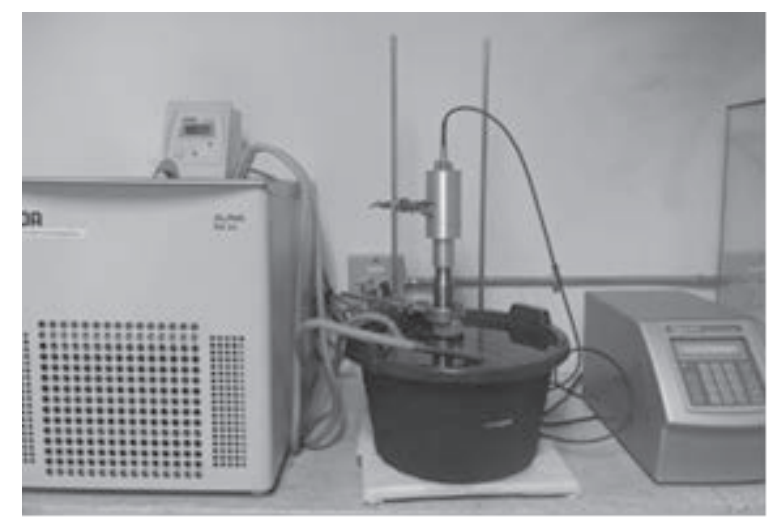

FIGURA 1. MONTAJE EXPERIMENTAL PARA LA PREPARACIÓN DE NANOFLUIDOS POR EL MÉTODO DE DOS PASOS 
Cacua, et al. / Estudio de los factores que afectan la estabilidad de nanofluidos...

TABLA 1. FACTORES Y NIVELES DEL DISEÑO EXPERIMENTAL

\begin{tabular}{|c|c|c|}
\hline Factores & Nivel inferior $(-1)$ & Nivel superior $(+1)$ \\
\hline A: Tipo de nanopartícula & $\mathrm{Al}_{2} \mathrm{O}_{3}$ & $\mathrm{TiO}_{2}$ \\
\hline B: Concentración de nanopartículas \%p/p & 0,1 & 0,5 \\
\hline C: Tipo de surfactante & $\mathrm{SDBS}$ & $\mathrm{CTAB}$ \\
\hline D: Concentración surfactante & $1 \mathrm{CMC}$ & $2 \mathrm{CMC}$ \\
\hline E: Amplitud de ultrasonido & $30 \%$ & $50 \%$ \\
\hline F: Tiempo de ultrasonido & $10 \mathrm{~min}$ & $20 \mathrm{~min}$ \\
\hline
\end{tabular}

TABLA 2. MATRIZ DE DISEÑO EXPERIMENTAL

\begin{tabular}{|c|c|c|c|c|c|c|c|}
\hline 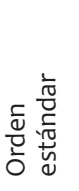 & 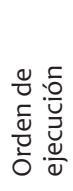 & 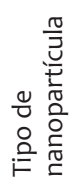 & 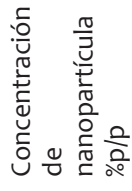 & 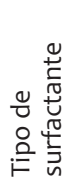 & 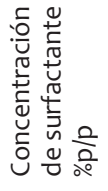 & 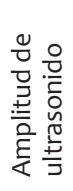 & 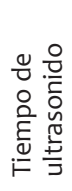 \\
\hline 7 & 1 & -1 & 1 & -1 & -1 & 1 & 1 \\
\hline 15 & 2 & 1 & 1 & 1 & -1 & 1 & -1 \\
\hline 2 & 3 & -1 & -1 & 1 & 1 & 1 & -1 \\
\hline 12 & 4 & -1 & 1 & 1 & 1 & -1 & 1 \\
\hline 5 & 5 & 1 & -1 & 1 & 1 & -1 & -1 \\
\hline 13 & 6 & 1 & 1 & -1 & -1 & -1 & 1 \\
\hline 4 & 7 & 1 & -1 & -1 & 1 & 1 & 1 \\
\hline 9 & 8 & 1 & 1 & 1 & 1 & 1 & 1 \\
\hline 10 & 9 & 1 & 1 & -1 & 1 & -1 & -1 \\
\hline 6 & 10 & 1 & -1 & 1 & -1 & -1 & 1 \\
\hline 16 & 11 & -1 & -1 & 1 & -1 & 1 & 1 \\
\hline 1 & 12 & -1 & -1 & -1 & 1 & -1 & 1 \\
\hline 3 & 13 & -1 & -1 & -1 & -1 & -1 & -1 \\
\hline 8 & 14 & -1 & 1 & -1 & 1 & 1 & -1 \\
\hline 14 & 15 & -1 & 1 & 1 & -1 & -1 & -1 \\
\hline 11 & 16 & 1 & -1 & -1 & -1 & 1 & -1 \\
\hline
\end{tabular}




\subsection{Evaluación de la estabilidad de los nanofluidos}

La evaluación de la estabilidad se realizó teniendo en cuenta la diferencia entre la conductividad térmica del nanofluido inmediatamente después de preparado $\left(K_{1}\right)$ y la conductividad térmica ocho días después $\left(K_{2}\right)$. Un valor de cero indica un nanofluido estable. La ecuación (1) muestra el cálculo de la estabilidad:

$$
\text { Estabilidad }=\left|\frac{K_{1}-K_{2}}{K_{1}}\right|
$$

\section{Resultados}

Para determinar los efectos principales y los efectos de las interacciones entre los factores sobre la estabilidad de los nanofluidos se utilizó el diagrama de Pareto y el análisis de varianza ANOVA. En el diagrama de Pareto se ordenan los efectos en orden decreciente de importancia en la variabilidad de la respuesta obtenida y el ANOVA separa la variación total en las partes con las que contribuye cada fuente de variación en el experimento ${ }^{[20]}$. Los cálculos fueron realizados con un nivel de significancia del $5 \%(\alpha=0,05)$. El valor $\mathrm{p}$ es la significancia observada, es decir la relación entre los cuadrados medios debidos a los tratamientos y los cuadrados medios debido al error, si $\mathrm{p}<\mathrm{a}$ el efecto o interacción es significativo.

\subsection{Diagrama Pareto}

El diagrama de Pareto muestra los efectos estimados y las interacciones ordenados desde el mayor hasta el menor, utilizando columnas. En la FIGURA 2 se muestran los efectos significativos y no significativos estadísticamente en la variación de la conductividad térmica. La línea vertical punteada indica cuán grande debe ser un efecto para ser estadísticamente significativo. Como se puede observar solo la interacción entre el tipo de nanopartícula y el tipo de surfactante tiene un efecto significativo sobre la variación de la conductividad térmica. Al combinar una nanopartícula específica con un determinado tipo de surfactante, ya sea iónico, catiónico o no iónico, este influencia el tipo de interacción que se presenta entre las nanopartículas, así como también, las propiedades térmicas e hidrodinámicas del nanofluido [21]. 
Para refinar el análisis, se aplicó el método de la curvatura sugerido por Gutiérrez y de la Vara ${ }^{[20]}$, en el cual se repite el análisis sólo con aquellos factores e interacciones que forman una tendencia curva en el diagrama de Pareto. Estos factores e interacciones son AC, F, D, AD y AF. Con esta elección, se logró obtener un total de 10 grados de libertad en el error. La interacción $\mathrm{ABF}$ no se tomó en cuenta debido a que es una interacción de tres factores, la cual no es significativa según los supuestos del diseño factorial fraccionado. Es posible que la aparición de esta interacción dentro de los primeros lugares en el diagrama de Pareto se deba a un efecto marcado del factor $\mathrm{F}$ o de la interacción AF. Es por esta razón que en el nuevo análisis se incluye la interacción AF, aun cuando esta no está definida completamente dentro de la concavidad superior.

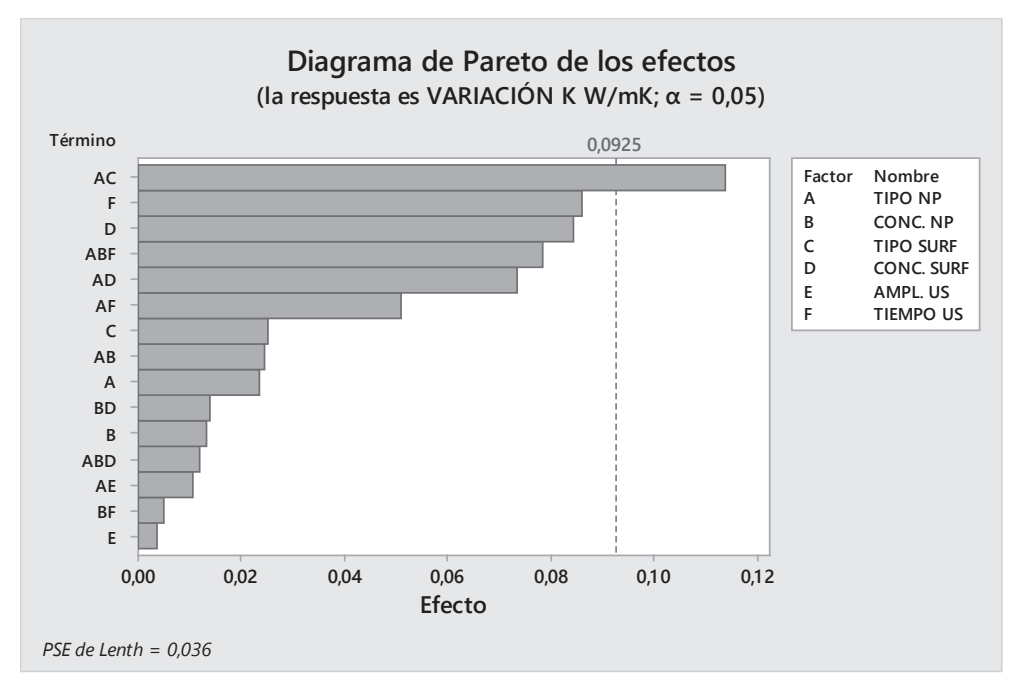

FiguRA 2. DIAGRAMA PARETO DE LOS EFECTOS EN LA VARIACIÓN

DE LA CONDUCTIVIDAD TÉRMICA

En la FIGURA 3 se muestra el diagrama de Pareto para la variación de la conductividad térmica incluyendo solamente los factores e interacciones AC, $F, D, A D$ y AF. En este caso se observa que los factores $F$ (tiempo de ultrasonido) y $D$ (concentración de surfactante), al igual que las interacciones $A C$ (tipo de nanopartícula y tipo de surfactante) y AD (tipo de nanopartícula y 
concentración de surfactante) resultaron tener un efecto significativo estadísticamente sobre la variación de la conductividad térmica del nanofluido.

\subsection{Análisis de varianza ANOVA}

Los resultados obtenidos en el diagrama de Pareto se confirman en el análisis de ANOVA que se muestra en la Tabla 3, donde se puede observar que las fuentes de variación AC, F, D, y AD tuvieron valores p de 0,003; 0,015; 0,016 y 0,031; respectivamente, menores que el nivel de significancia de 0,05. En la Tabla 3, columna 5 se muestra el estadístico de prueba $\mathrm{F}$, un valor de $\mathrm{F}$ grande indica un efecto estadístico significativo del factor o su interacción sobre la variable respuesta.

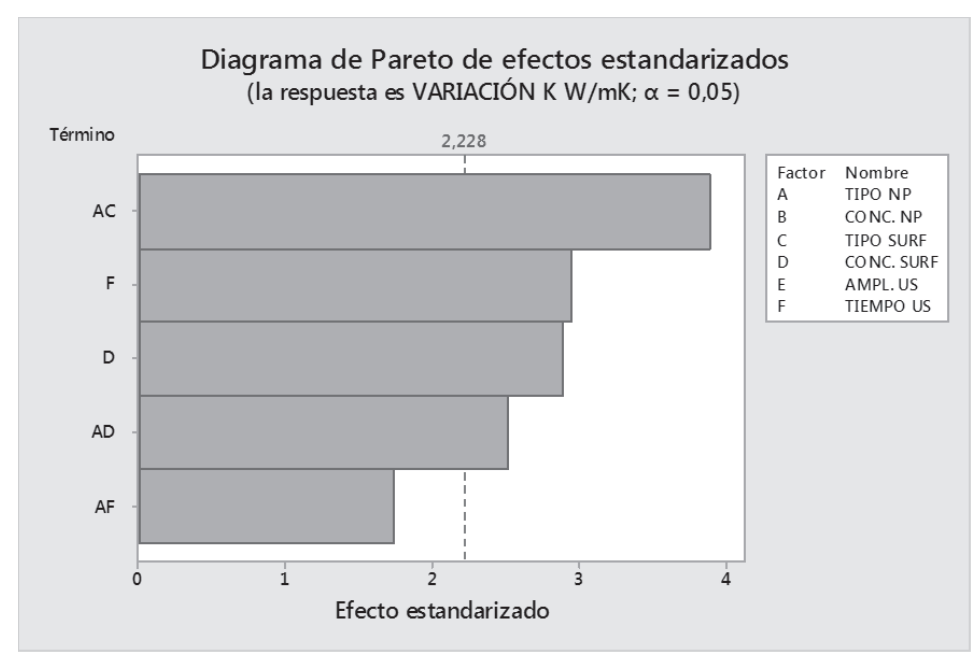

FIGURA 3. DIAGRAMA PARETO DE LOS EFECTOS EN LA VARIACIÓN

DE LA CONDUCTIVIDAD TÉRMICA

TABLA 3. ANÁLISIS ANOVA DEL DISEÑO FACTORIAL FRACCIONADO CON LOS FACTORES AC, F, D, AD Y AF

\begin{tabular}{|l|l|l|l|l|l|}
\hline Fuente & GL & SC Ajustado & MC Ajustado & F & P \\
\hline Modelo & 5 & 0,1416 & 0,0283 & 8,2800 & 0,0030 \\
\hline Lineal & 2 & 0,0580 & 0,0290 & 8,4700 & 0,007 \\
\hline Conc. Surf. & 1 & 0,0284 & 0,0284 & 8,2900 & 0,0160 \\
\hline
\end{tabular}


CONTINUACIÓN TABLA 3. ANÁLISIS ANOVA DEL DISEÑO FACTORIAL FRACCIONADO CON LOS FACTORES AC, F, D, AD Y AF

\begin{tabular}{|l|l|l|l|l|l|}
\hline Fuente & GL & SC Ajustado & MC Ajustado & F & p \\
\hline Tiempo US & 1 & 0,0296 & 0,0296 & 8,6400 & 0,0150 \\
\hline Int. Dobles & 3 & 0,0837 & 0,0279 & 8,1500 & 0,0050 \\
\hline Tipo NP - Tipo Surf. & 1 & 0,0518 & 0,0517 & 15,1200 & 0,0030 \\
\hline Tipo NP - Conc. Surf. & 1 & 0,0216 & 0,0216 & 6,3100 & 0,0310 \\
\hline Tipo NP -Tiempo US. & 1 & 0,0103 & 0,0103 & 3,0100 & 0,1130 \\
\hline Error & 10 & 0,0342 & 0,0034 & & \\
\hline
\end{tabular}

GL: GRADOS DE LIBERTAD; SC: SUMA DE CUADRADOS; MC: CUADRADOS MEDIOS; F: ESTADÍSTICO DE PRUEBA; P: SIGNIFICANCIA OBSERVADA

En la Tabla 4 se muestra la estructura de alias para los factores e interacciones seleccionados, los alias son efectos que son indistinguibles. Se nota claramente que los factores principales $D$ y $F$ solo están aliados con interacciones de tres y cinco factores, por lo que se puede decir con certeza que los efectos de esta estructura de alias son atribuibles exclusivamente a estos factores, debido a la poca significancia práctica que tiene tienen las interacciones de tres o más factores. Las interacciones $A C$ y $A D$ están aliadas con las interacciones dobles $\mathrm{BE}$ y $\mathrm{EF}$, por lo que no puede distinguirse su efecto único por encima de estas interacciones.

TABLA 4. ESTRUCTURA DE ALIAS DE LOS FACTORES E INTERACCIONES SELECCIONADAS

\begin{tabular}{|c|}
\hline$I+A B C E+A D E F+B C D F$ \\
\hline$D+A E F+B C F+A B C D E$ \\
\hline$F+A D E+B C D+A B C E F$ \\
\hline$A C+B E+A B D F+C D E F$ \\
\hline$A D+E F+A B C F+B C D E$ \\
\hline$A F+D E+A B C D+B C E F$ \\
\hline
\end{tabular}

El hecho de que la interacción AC (tipo de nanopartícula y tipo de surfactante) resulte significativo es consecuente con el tipo de interacción quí- 
mica que se presenta en este tipo de sistemas, ya que al combinar una nanopartícula específica con un surfactante se presentan una serie de efectos de atracción y repulsión entre las nanopartículas que llevan a una aglomeración o separación de las mismas, trayendo como consecuencia cambios en la conductividad térmica del nanofluido ${ }^{[22]}$. No obstante, esta interacción está aliada con la interacción BE (concentración de nanopartículas y amplitud de la sonicación), la cual también explicaría un cambio en la conductividad térmica, dado que los aumentos en concentración de nanopartículas conllevan aumentos en conductividad térmica y la amplitud de la sonicación afecta la dispersión de las nanopartículas.

Por lo tanto, no se puede concluir con certeza a cuál de estas interacciones dobles aliadas se puede atribuir el efecto observado sobre la variación de la conductividad. Esta misma ambivalencia se presenta entre interacciones AD (tipo de nanopartícula y concentración de surfactante) y EF (amplitud y tiempo de sonicación), dado que la conductividad térmica depende de la interacción del surfactante con el tipo de nanopartícula y con el fluido base, pero también se debe tener en cuenta que la amplitud y el tiempo de sonicación en conjunto afectan la dispersión de las nanopartículas, influyendo sobre la conductividad térmica del nanofluido. Con estos resultados preliminares y la resolución de este diseño, no es posible concluir cuáles efectos o sus interacciones tienen mayor influencia sobre la variación de la conductividad térmica con el tiempo y establecer un modelo estadístico predictivo de esta variación.

\section{Conclusiones}

Se utilizó un diseño factorial fraccionado de resolución IV con el fin de identificar preliminarmente el efecto de seis factores y/o sus interacciones en la variación de la conductividad térmica de nanofluidos, como indicativo de estabilidad.

El tiempo de ultrasonido y la concentración de surfactante tienen una influencia significativa sobre la variación de la conductividad térmica del nanofluido. Sin embargo, es necesario evaluar con mayor profundidad dicho efecto con el fin de obtener valores óptimos de estos factores que permitan minimizar la variación en la conductividad térmica con el tiempo. 
La interacción del tipo de nanopartícula con el tipo y la concentración de surfactante influyen significativamente sobre la variación de la conductividad térmica, aunque estos efectos están confundidos con otros factores determinantes de la estabilidad de un nanofluido como son la amplitud y el tiempo de sonicación, por lo que no se puede concluir sobre cual efecto es más significativo en la variación de la conductividad térmica.

Es necesario aplicar el diseño factorial fraccionado como herramienta de análisis preliminar teniendo en cuenta otras variables respuesta, tales como variación en la absorbancia, potencial zeta y dispersión de luz dinámica, con el fin de confirmar cuál factor o sus interacciones tienen un efecto significativo en la estabilidad de los nanofluidos y poder confirmar los resultados obtenidos en este primer estudio.

\section{Agradecimientos}

Los autores desean expresar su agradecimiento a COLCIENCIAS por la financiación del proyecto (1118-669-46092 СTO: 126-2015) dentro del cual se enmarca esta investigación.

\section{Referencias}

[1] R. Kamatchi and S. Venkatachalapathy, "Parametric study of pool boiling heat transfer with nanofluids for the enhancement of critical heat flux: A review," Int. J. Therm. Sci., vol. 87, pp. 228-240, Jan. 2015.

[2] S. K. Das, S. U. S. Choi, and H. E. Patel, "Heat Transfer in Nanofluids-A Review," Heat Transf. Eng., vol. 27, no. 10, pp. 3-19, Dec. 2006.

[3] S. Choi and J. Eastman, "Enhancing thermal conductivity of fluids with nanoparticles," Int. Mech. Eng. Congr. Expo., pp. 12-17, 1995.

[4] J. Sarkar, P. Ghosh, and A. Adil, "A review on hybrid nanofluids: Recent research, development and applications," Renew. Sustain. Energy Rev., vol. 43, pp. 164-177, Mar. 2015.

[5] C. Pang, J. W. Lee, and Y. T. Kang, "Review on combined heat and mass transfer characteristics in nanofluids," Int. J. Therm. Sci., vol. 87, pp. 49-67, Jan. 2015.

[6] L. Yang, K. Du, X. S. Zhang, and B. Cheng, "Preparation and stability of $\mathrm{Al}_{2} \mathrm{O}_{3}$ nano-particle suspension of ammonia-water solution," Appl. Therm. Eng., vol. 31, no. 17-18, pp. 3643-3647, Dec. 2011. 
[7] W. Yu and H. Xie, "A Review on Nanofluids: Preparation, Stability Mechanisms, and Applications," J. Nanomater., vol. 2012, pp. 1-17, 2012.

[8] N. A. C. Sidik, H. a. Mohammed, O. a. Alawi, and S. Samion, "A review on preparation methods and challenges of nanofluids," Int. Commun. Heat Mass Transf., vol. 54, pp. 115-125, May 2014.

[9] J. Philip and P. D. Shima, "Thermal properties of nanofluids.," Adv. Colloid Interface Sci., vol. 183-184, pp. 30-45, Nov. 2012.

[10] L. Fedele, L. Colla, S. Bobbo, S. Barison, and F. Agresti, "Experimental stability analysis of different water-based nanofluids.," Nanoscale Res. Lett., vol. 6, no. 1, p. 300, Jan. 2011.

[11] Babita, S. K. Sharma, and G. S. Mital, "Preparation and evaluation of stable nanofluids for heat transfer application: A review," Exp. Therm. Fluid Sci., vol. 79, pp. 202-212, 2016.

[12] C. Anushree and J. Philip, "Assessment of Long term Stability of Aqueous Nanofluids Using Different Experimental Techniques,” J. Mol. Liq., vol. 222, pp. 350-358, 2016.

[13] X. Li, Y. Chen, S. Mo, L. Jia, and X. Shao, "Effect of surface modification on the stability and thermal conductivity of water-based SiOz-coated graphene nanofluid," Thermochim. Acta, vol. 595, pp. 6-10, Nov. 2014.

[14] N. Nikkam, “Engineering Nanofluids for Heat Transfer Applications," Royal Institute of Technology (KTH), 2014.

[15] M. E. Meibodi, M. Vafaie-Sefti, A. M. Rashidi, A. Amrollahi, M. Tabasi, and H. S. Kalal, "The role of different parameters on the stability and thermal conductivity of carbon nanotube/water nanofluids," Int. Commun. Heat Mass Transf., vol. 37, no. 3, pp. 319-323, 2010.

[16] X. Wang, X. Li, and S. Yang, "Influence of pH and SDBS on the Stability and Thermal Conductivity of Nanofluids," no. 16, pp. 2684-2689, 2009.

[17] A. Ghadimi, R. Saidur, and H. S. C. Metselaar, "A review of nanofluid stability properties and characterization in stationary conditions," Int. J. Heat Mass Transf., vol. 54, no. 17-18, pp. 4051-4068, Aug. 2011.

[18] D. Montgomery, Design and analysis of experiments, Eight edit. Arizona: John Wiley \& Sons Ltd., 2013.

[19] Minitab Inc, “MINITAB versión 17.".

[20] H. Gutiérrez and R. De la Vara, Análisis y diseño de experimentos, Segunda ed. McGraw-Hill, 2008.

[21] R. V. Pinto and F. A. S. Fiorelli, "Review of the Mechanisms Responsible for Heat Transfer Enhancement Using Nanofluids," Appl. Therm. Eng., vol. 108, pp. 720-739, 2016.

[22] C. Pang, J.-Y. Jung, and Y. T. Kang, "Aggregation based model for heat conduction mechanism in nanofluids," Int. J. Heat Mass Transf., vol. 72, pp. 392-399, May 2014. 